02

\title{
Спектральное исследование фотодинамических процессов в системе $\mathrm{C}_{60}-(\boldsymbol{\mu}$-метилпирролидон)-кислород
}

\author{
(C) И.М. Кисляков ${ }^{1,2}$, И.М. Белоусова ${ }^{1}$, В.М. Киселев ${ }^{1}$, И.В. Багров ${ }^{2}$, А.М. Стародубцев ${ }^{1}$, Tianju Zhang ${ }^{2,3}$, \\ Jun Wang ${ }^{2,3}$ \\ ${ }^{1}$ Государственный оптический институт им. С.И. Вавилова, \\ 199034 Санкт-Петербург, Россия \\ ${ }^{2}$ Shanghai Institute of Optics and Fine Mechanics, Chinese Academy of Sciences, \\ 201800 Shanghai, China \\ ${ }^{3}$ Center of Materials Science and Optoelectronic Engineering, University of Chinese Academy of Sciences, \\ 100049 Beijing, China \\ e-mail: iv.kis@mail.ru
}

Поступила в редакцию 30.06.2021 г.

В окончательной редакции 13.08.2021 г.

Принята к публикации 17.08.2021 г.

Исследованы процессы фотовозбуждения и переноса энергии в воздухонасыщенном растворе фуллерена $\mathrm{C}_{60}$ в $H$-метилпирролидоне. При фемтосекундной лазерной накачке на длине волны $\lambda_{\mathrm{p}}=520 \mathrm{~nm}$ получены спектры переходного поглощения в области $470-750 \mathrm{~nm}$. Наблюдается широкополосное поглощение при $\lambda>650 \mathrm{~nm}$ в результате агрегации молекул фуллерена, характеризующееся коротким периодом затухания пикосекундного масштаба. Анализ временных зависимостей указывает на наличие нескольких процессов. В рамках предложенной интерпретации мы констатируем значительное сокращение времени внутрисистемного синглет-триплетного переноса при увеличении размеров нанокластеров, в то время как быстрое тушение синглетного состояния меняется мало. Квантовый выход триплетного состояния может при этом возрастать, достигая 0.14. Измерены спектры фосфоресценции синглетного кислорода (СК) с максимумом на $\lambda=1276 \mathrm{~nm}$ при светодиодном возбуждении на различных длинах волн в диапазоне $370-625 \mathrm{~nm}$. Определены время жизни и константа скорости тушения СК в $H$-метилпирролидоне. Совокупный квантовый выход триплетного фуллерена и СК в $\boldsymbol{H}$-метилпирролидоне оценивается в 0.74 . Построены спектры эффективности фотосенсибилизации синглетного кислорода в системе $\mathrm{C}_{60}$-(н-метилпирролидон)-кислород, указывающие на возрастающую роль крупных нанокластеров в этом процессе при старении раствора.

Ключевые слова: фуллерен, $\boldsymbol{H}$-метилпирролидон, нанокластеры, молекулярные комплексы, внутрисистемный перенос, триплетное состояние, синглетный кислород, фотосенсибилизация, фосфоресценция, квантовый выход.

DOI: $10.21883 / \mathrm{OS} .2021 .12 .51736 .2429-21$

\section{Введение}

Работа посвящается памяти профессора М.О. Буланина - одного из создателей школы спектроскопии атомно-молекулярных систем в Санкт-Петербургском государственном университете, с которым И.М.Б. училась и под руководством которого И.М.К. осваивал профессию. Авторы от всего сердца присоединяются к коллегам, отмечающим его 90-летие и вспоминают его вклад в развитие этой области знаний. По поводу темы настоящей работы авторы отмечают, что профессор Буланин, начиная с самого открытия фуллеренов, интересовался их структурой и свойствами и своими увлекательными лекциями прививал этот интерес ученикам.

Молекула бакминстерфуллерена является уникальной во многих отношениях [1,2], и своими уникальными оптическими свойствами она обязана делокализованным и полностью заполненным $\pi$-электронным оболочкам при сохранении высокой симметрии молекулы, близкой к сферической. Их размер и подвижность определяют большие величины поляризуемостей, приближающие фуллерен к красителям по величине сечений линейного и нелинейного оптического поглощения. Высокая симметрия, относящаяся к икосаэдрической группе $I_{h}$, способствует вырождению нижних электронных состояний, на которые сильно влияют колебания молекулярного каркаса, вызывая интенсивные ян-теллеровские и герцберг-теллеровские эффекты. Они в значительной степени определяют спектральные особенности поглощения и люминесценции фуллерена в видимой и ближней ИК областях спектра [3].

Как часто наблюдается и в ряду красителей, делокализованные $\pi$-электроны фуллерена со слабым отталкиванием создают благоприятные условия для синглет-триплетного взаимодействия [4], проявляющегося в преимущественном заселении триплетного состояния фуллерена при оптическом возбуждении и, следом, в резонансном переносе энергии на состояние ${ }^{1} \Sigma_{g}^{+}$молекулярного кислорода при столкновительном взаимодей- 


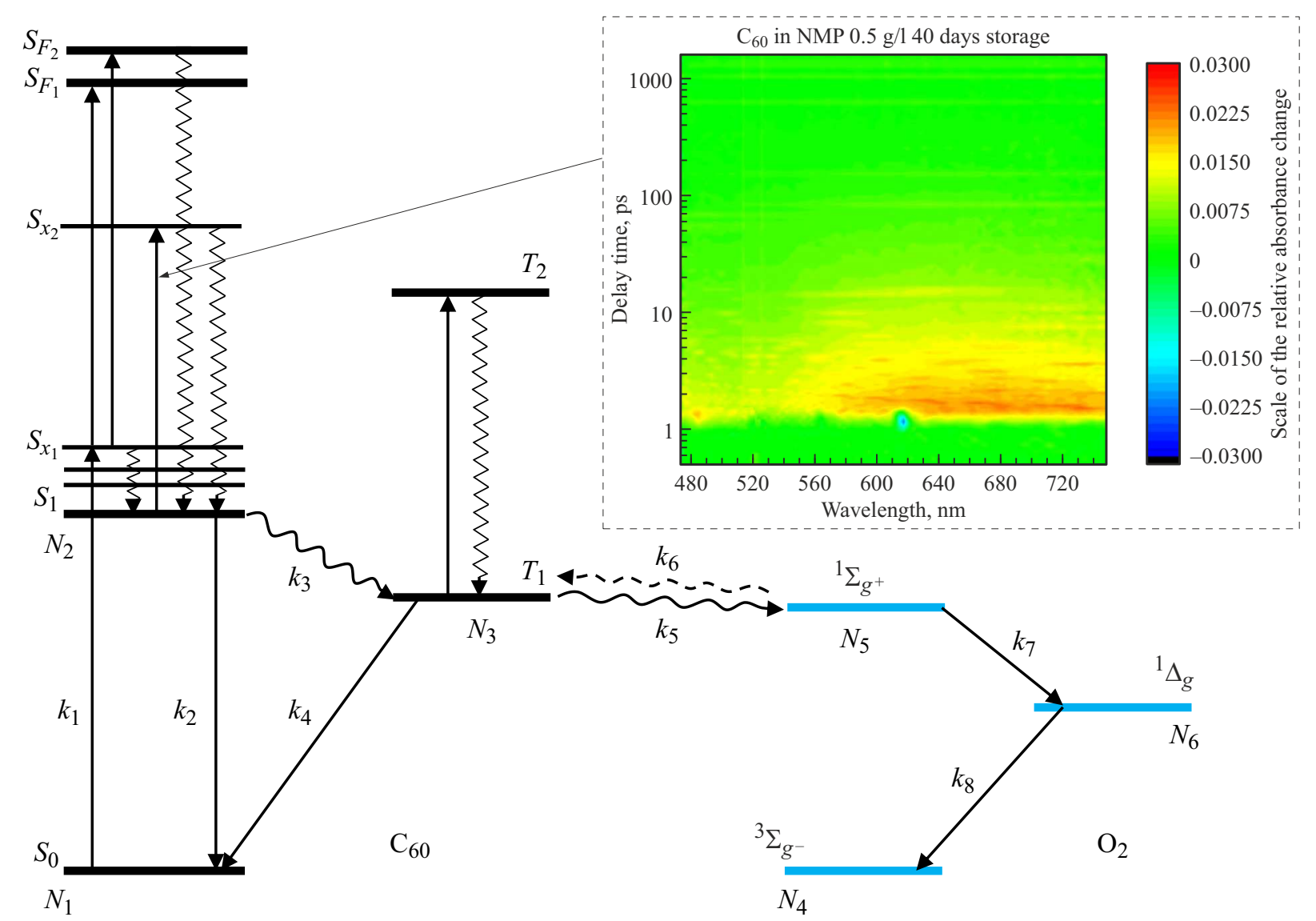

Рис. 1. Схема возбуждения энергетических уровней системы $\mathrm{C}_{60}-\mathrm{O}_{2}$. Вставка: спектр переходного поглощения 40-дневного раствора $\mathrm{C}_{60}$ в NMP.

ствии (рис. 1). Высокий квантовый выход этого процесса делает фуллерен одним из лучших фотосенсибилизаторов синглетного кислорода (СК) [4-6]. Перспективы его применения в качестве активного агента в фотодинамической терапии поверхностных опухолей и инактивации патогенов в биологических жидкостях широко изучались $[7,8]$. Ничтожно малая растворимость фуллерена в водных системах является, с одной стороны, благоприятным фактором, облегчающим его удаление после фотодинамического воздействия на биологическую систему, а с другой, фактором, снижающим эффективность фотосенсибилизации (ФС). Поэтому много усилий предпринималось для создания и исследования устойчивых золей на водной основе, сохраняющих ФС-свойства [9]. Для этого применялись как поверхностно-активные вещества [10], так и водно-мицеллярные коллоиды [11]. Применение обеих систем проблематично в сочетании с реальными биологическими жидкостями, что заставляет продолжать поиск подходящей системы.

Золь на основе $н$-метилпирролидона (NMP) давно привлекает исследователей [12-14] в связи с простотой получения, малыми размерами частиц фуллерена и соответственно хорошей временной устойчивостью, а также относительно низкой токсичностью этого растворителя. Хотя растворимость кислорода в NMP при температуре
$T=25^{\circ} \mathrm{C}(3.26 \mathrm{mmol} / \mathrm{l}[15])$ ниже, чем в органических растворителях $(9.88 \mathrm{mmol} / \mathrm{l}$ в толуоле и $12.4 \mathrm{mmol} / \mathrm{l}$ в четыреххлористом углероде $\left.\left(\mathrm{CCl}_{4}\right)[16]\right)$, это больше, чем в воде $(1.27 \mathrm{mmol} / \mathrm{l}[16])$, что дает повод рассмотреть вопросы ФС СК в этой среде.

Важным факторов, определяющим ФС СК, является внутрисистемный перенос $S_{1}-T_{1}$ (процесс, характеризующийся константой скорости $k_{3}$ на рис. $1, k_{3}=k_{\text {isc }}$ ). Ранее в работе [12] методом флэш-фотолиза были зарегистрированы переходные спектры триплет-триплетного поглощения $\left(T_{1}-T_{2}\right)$ и поглощения анион-радикала $\mathrm{C}_{60}^{\bullet-}$ в NMP с добавкой донора электрона. Положение максимума триплет-триплетного спектра на $740 \mathrm{~nm}$ оказалось приблизительно совпадающим с его максимумом в толуоле (747 nm [17]), а время жизни триплетного состояния $\left(\tau_{\mathrm{T}}=1 / k_{4} \sim 340 \mu \mathrm{s}\right)$ примерно в 330 раз меньше, чем в бензольном растворе такой же концентрации [18]. Авторы изучили механизм ФС анион-радикала $\mathrm{O}_{2}^{\bullet-}$, но не смогли зарегистрировать ФС СК. Был сделан вывод о том, что перенос заряда в системе препятствует переносу энергии.

В нашей работе, пользуясь чувствительной техникой, мы регистрируем процесс ФС СК в отсутствие доноров, анализируем его спектр, эффективность и скорость тушения СК растворителем. Мы также анализируем 
начальный этап фотодинамического переноса энергии скорости релаксации синглетных состояний и внутрисистемного переноса фуллерена с одновременным наблюдением возрастных изменений в системе $\mathrm{C}_{60}-\mathrm{NMP}-\mathrm{O}_{2}$.

\section{Методика эксперимента}

$\mathrm{B}$ исследованиях использовался фуллерен $\mathrm{C}_{60}$ производства ЗАО ИЛИП (Санкт-Петербург) хроматографически контролируемой чистоты $99.96 \%$ и продукт Sigma-Aldrich № $57250099.9 \%$ чистоты. Навеска фуллерена растворялась в NMP (Sigma-Aldrich № 328634, обезвоженный, 99.5\%), бензоле (Sigma-Aldrich № 401765, обезвоженный, 99.8\%) или четыреххлористом углероде (Sigma-Aldrich № 270652, 99.9\%) при непрерывном перемешивании в течение $3 \mathrm{~h}$ для получения соответствующей концентрации. Линейные спектры поглощения полученных растворов в ближней УФ и видимой областей записывались на двухлучевом спектрофотометре Shimadzu UV-3600 относительно чистых растворителей. Характерные спектры поглощения приведены на рис. 2. Они воспроизводят качественные изменения, наблюдавшиеся в растворах $\mathrm{C}_{60}$ в NMP и ранее [13].

ФС СК исследовалась по наблюдению его фосфоресценции в области $1268 \mathrm{~nm}$ (длина волны ${ }^{1} \Delta_{g}-{ }^{3} \Sigma_{g}$-перехода). Спектры фотолюминесценции (ФЛ) растворов регистрировались ИК спектрометром SDH-IV (SOLAR Laser Systems, Беларусь) из конической колбы объемом $25 \mathrm{ml}$ при возбуждении набором сверхъярких светодиодов с максимумами излучения на длинах волн $\lambda_{\mathrm{e}}=370$, $405,465,525,595$ и $625 \mathrm{~nm}$. Время экспозиции составляло $12 \mathrm{~s}$. Подробное техническое описание установки приводится в [19]. ФЛ-измерения производились со свежим раствором на следующий день после его приготовления и с тем же раствором спустя пять месяцев.

Временной профиль фосфоресценции СК измерялся германиевым фотодиодом с интерференционным светофильтром, пропускающим область $1270 \pm 5 \mathrm{~nm}$, при

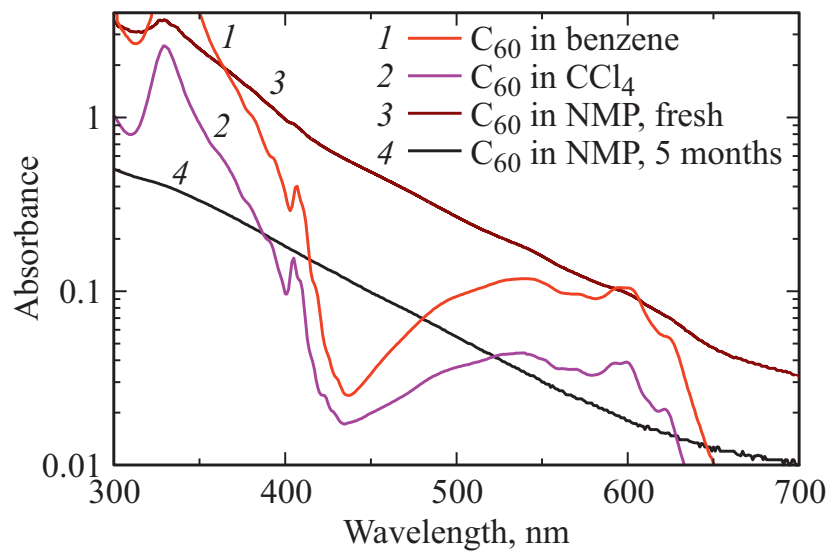

Рис. 2. Спектры поглощения растворов $\mathrm{C}_{60}$ в бензоле $(1 \mathrm{~g} / 1$, слой $1 \mathrm{~mm}), \mathrm{C}_{60}$ в $\mathrm{CCl}_{4}(0.1 \mathrm{~g} / \mathrm{l}$, слой $3 \mathrm{~mm}), \mathrm{C}_{60}$ в $\mathrm{NMP}(0.2 \mathrm{~g} / \mathrm{l}$, слой $3 \mathrm{~mm}$ ) - свежего (один день) и пятимесячного.
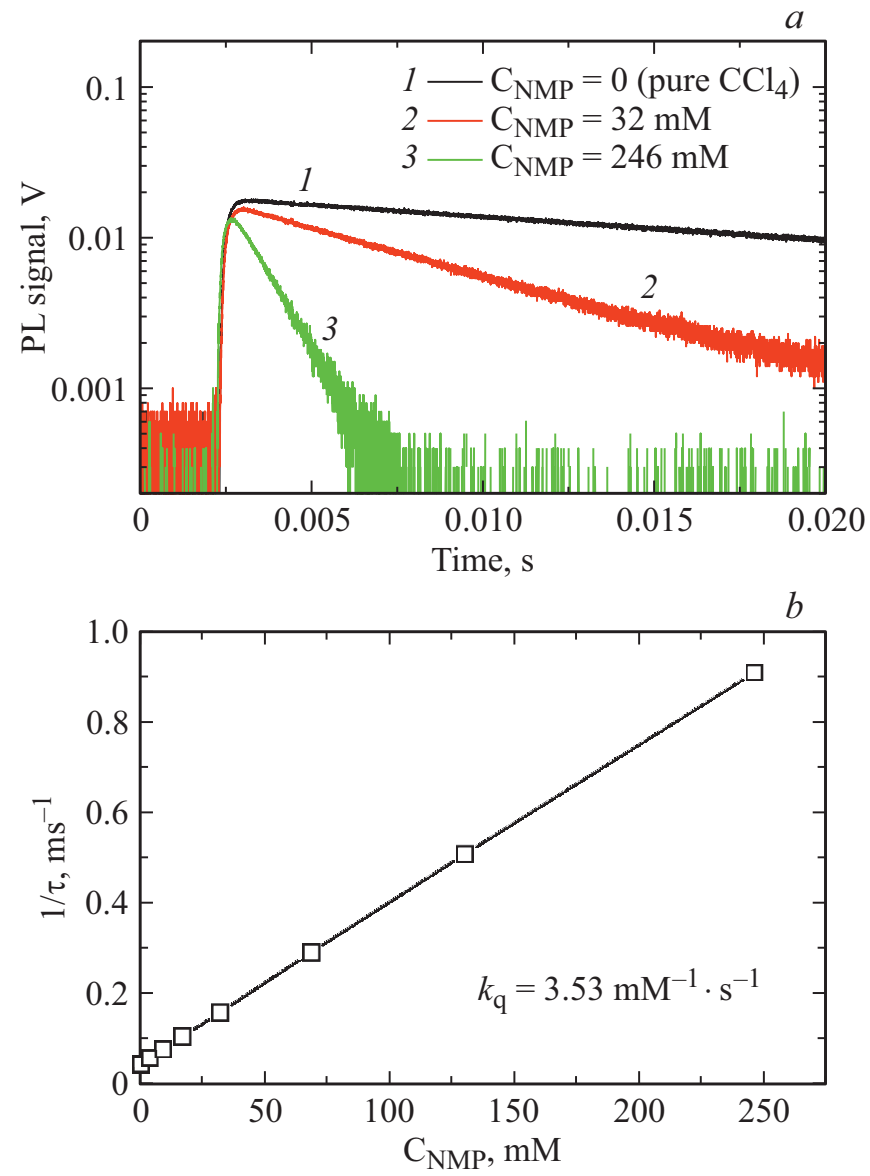

Рис. 3. Затухание сигнала фосфоресценции СК $(a)$ и рост коэффициента затухания $(b)$ с ростом концентрации NMP в растворе $\mathrm{C}_{60}$ в $\mathrm{CCl}_{4}$.

возбуждении растворов импульсной ксеноновой лампой с длительностью импульса $0.5 \mu \mathrm{s}$. Временные измерения имели цель определения константы скорости тушения СК $\left(k_{\mathrm{q}}=k_{8}\right.$ на рис. 1) применяемыми растворителями (главным образом NMP) и выполнялись на основе раствора $\mathrm{C}_{60}$ в $\mathrm{CCl}_{4}$, к которому последовательно добавлялся NMP в возрастающей концентрации. Данные растворители хорошо смешиваются, а присутствие NMP в небольших концентрациях относительно слабо влияло на концентрацию $\mathrm{C}_{60}$ в растворе за время измерений. Наблюдалось укорочение времени фосфоресценции СК c $\tau_{\mathrm{SO}}=25 \mathrm{~ms}$ в чистом $\mathrm{CCl}_{4}$ до $1.1 \mathrm{~ms}$ при $246 \mathrm{mmol} / 1$ $\mathrm{NMP}$, как показано на рис. 3, $a$, и рост экспоненциального коэффициента ее затухания с концентрацией NMP (рис. $3, b)$, линейная аппроксимация которого дает значение $k_{\mathrm{q}}=3.53 \pm 0.01 \mathrm{mmol}^{-1} \cdot 1 \cdot \mathrm{s}^{-1}$, что соответствует времени жизни СК $\tau_{\mathrm{SO}}(\mathrm{NMP})=27.2 \mu \mathrm{s}$.

Спектры переходного поглощения исследовались на установке, собранной с использованием второй гармоники (длина волны $\lambda_{\mathrm{p}}=520 \mathrm{~nm}$ ) фемтосекундного лазера Spectra Physics Spirit $(350 \mathrm{fs}, 1 \mathrm{kHz}$ и $40 \mathrm{~mJ}$ на импульс) в качестве источника накачки, часть энергии которой использовалась для генерации суперконтину- 
ального пробного луча с шириной спектра $450-750 \mathrm{~nm}$ с помощью сапфирового стекла. Луч накачки амплитудно модулировался с частотой $500 \mathrm{~Hz}$. Пробный луч проходил моторизированную линию задержки с длиной хода $1.7 \mathrm{~ns}$ и временным разрешением $12.5 \mathrm{fs}$ и после прохождения образца регистрировался на быстром спектрометре с волоконным входом. Инкремент поглощения рассчитывался как $\Delta A=1-I_{\text {pump on }} / I_{\text {pump off. }}$

\section{Результаты и обсуждение}

\section{Кинетика возбужденных состояний}

Спектрально-временная карта переходного поглощения приведена для наглядности на вставке на рис. 1 для 40-дневного раствора $\mathrm{C}_{60}$ в NMP. На графиках $a, b$ и $c$ на рис. 4 приводятся переходные спектры, соответствующие растворам $\mathrm{C}_{60}-$-бензол, свежему $\mathrm{C}_{60}-\mathrm{NMP}(3-5 \mathrm{~h})$ и 40-дневному $\mathrm{C}_{60}-\mathrm{NMP}$ при нескольких временах задержки пробного импульса. В бензольном растворе хорошо виден рост триплет-триплетного поглощения (переход $T_{1}-T_{2}$ ) с максимумом на $740 \mathrm{~nm}$, происходящий одновременно со спадом менее заметного в данной системе синглет-синглетного поглощения (переход $\left.S_{1}-S x_{2}\right)$ в зеленой области спектра. В NMP синглет-синглетное поглощение уширяется и сдвигается в длинноволновую область при $\lambda>600 \mathrm{~nm}$, причем со временем это смещение усиливается, а спектр становится более компактным, обнаруживая средневзвешенный максимум в районе $680 \mathrm{~nm}$. Рост триплет-триплетного поглощения при этом не наблюдается, поскольку триплетное состояние сильно тушится фуллереном в плотной упаковке кластеров. Однако из работы [12] известно, что оно имеет место в этой системе, и его максимум по-прежнему приходится на $740 \mathrm{~nm}$. Любопытно, что на переднем фронте сплошного поглощения наблюдаются узкие провалы насыщенного поглощения на длинах волн $617\left(h v_{1}=2.01 \mathrm{eV}\right)$ и $564 \mathrm{~nm}\left(h v_{2}=2.20 \mathrm{eV}\right)$, которые по суммарной частоте $\left(h v_{\mathrm{p}}+h v_{1}\right.$ и $\left.h v_{\mathrm{p}}+h v_{2}\right)$ оказываются резонансными $F_{1}$ - и $F_{2}$-переходам в твердофазном фуллерене [3]. Их насыщение происходит за счет насыщения уровня $S x_{1}$ (аналогично блокировке Паули в твердом теле) и релаксирует вместе с релаксацией $S x_{1}$ в $S_{1}$. На карте (рис. 1) хорошо видно, что соответствующие этому насыщению провалы в основном исчезают еще до старта наведенного поглощения, которое, таким образом, происходит с уровня $S_{1}$.

Временная зависимость переходного поглощения приведена для тех же растворов на графиках $d, e, f$ на рис. 4 для ряда длин волн как в области линейного поглощения $(475,532 \mathrm{~nm})$, так и под границей линейного поглощения $(600-740 \mathrm{~nm})$. Аппроксимации экспериментальных зависимостей показаны на графиках более светлыми гладкими кривыми для $475 \mathrm{~nm}$ (или $532 \mathrm{~nm}$ в случае $\mathrm{C}_{60}$ в бензоле) и $740 \mathrm{~nm}$. В случае раствора $\mathrm{C}_{60}$ в бензоле передний фронт наведенного поглощения довольно пологий и требует учета времени нарастания наведенного поглощения $\tau_{\mathrm{R}}$. Его спад аппроксимируется всего лишь одной экспонентой с временем жизни $\tau_{0}$ :

$$
\begin{aligned}
& \Delta A(t)=A_{1} e^{-\frac{t}{\tau_{0}}}\left(1-e^{-\frac{t}{\tau_{\mathrm{R}}}}\right) \\
& \times\left[1+\operatorname{erfc}\left(\frac{\tau_{\mathrm{p}}}{\sqrt{2} \tau_{\mathrm{R}}}-\frac{t}{\sqrt{2} \tau_{\mathrm{p}}}\right)\right]+A_{2}\left(1-e^{-\frac{t}{\tau_{0}}}\right) .
\end{aligned}
$$

Здесь множитель в квадратных скобках учитывает корреляцию переднего фронта сигнала поглощения с временным профилем импульса накачки длительностью $\tau_{\mathrm{p}}$ в приближении его гауссовой формы [20]. Время, характеризующее спад синглет-синглетного поглощения,

$$
\tau_{0}=\frac{1}{k_{\mathrm{r}}+k_{\mathrm{nr}} N_{1}+k_{\mathrm{isc}}},
$$

где $k_{\mathrm{r}}$ характеризует радиационный, а $k_{\mathrm{nr}}-$ нерадиационный (столкновительный) распад уровня $S_{1} ; N_{1}-$ концентрация фуллерена в основном состоянии. Таким образом, $k_{\mathrm{r}}+k_{\mathrm{nr}} N_{1}=k_{2}$ на рис. 1 . При анализе мономолекулярных растворов фуллерена принято пренебрегать $k_{\mathrm{r}}$ и $k_{\mathrm{nr}}$ как более медленными процессами [4]. Поэтому время $\tau_{0}$ должно одновременно характеризовать нарастание триплет-триплетного поглощения в районе $750 \mathrm{~nm}$ (второй член в $\left.(1), A_{2}>0\right)$, т. е. $\tau_{0}=\tau_{\text {isc }}=1 / k_{\text {isc }}$. Хотя наша установка не позволяет прописать максимум триплет-триплетного поглощения, но одновременная аппроксимации экспериментальных кривых на двух длинах волн позволяет определить это значение: $\tau_{\text {isc }}=3.5 \mathrm{~ns}$. Для раствора $\mathrm{C}_{60}$ в толуоле это время несколько ниже: $\tau_{\text {isc }}=1.2 \mathrm{~ns}[17]$, но имеет тот же порядок величины. Различие стоит отнести к разной морфологии „рыхлых“, фрактальных агрегатов [21], всегда существующих даже в (псевдо)мономолекулярных растворах, взаимодействие в которых влияет на это время в соответствии с (2).

Растворы $\mathrm{C}_{60}$ в NMP изучались при интенсивности накачки, в 10 раз большей $\left(I_{\mathrm{p}}=23 \mathrm{GW} / \mathrm{cm}^{2}\right)$, поэтому амплитуды наведенного синглет-синглетного поглощения заметно больше, что позволяет более подробно рассмотреть начальные фазы процессов релаксации, характеризуемые несколькими экспонентами:

$$
\Delta A(t)=\sum_{i=1}^{n} A_{i} e^{\frac{1}{\tau_{i}}}\left[1+\operatorname{erfc}\left(\frac{\tau_{\mathrm{p}}}{\sqrt{2} \tau_{i}}-\frac{t}{\sqrt{2} \tau_{\mathrm{p}}}\right)\right] .
$$

Требуется, как минимум, шесть экспонент $(n=6)$ для удовлетворительного описания кривых затухания. Из рисунков видно, что спад поглощения приблизительно одинаков во всей области наблюдаемой полосы поглощения $(\lambda>600 \mathrm{~nm})$. Времена релаксации, определенные из наших данных, приведены в таблице. Первая экспонента описывает насыщенное поглощение $\left(A_{1}<0\right)$, которое релаксирует за очень короткое время $\tau_{1}=140-210 \mathrm{fs}$ и вместе с $\tau_{2}$ характеризует релаксацию $S x_{1}$ состояния в $S_{1}$.

Следует отметить, что большое количество параметров в аппроксимации сказывается на точности их 

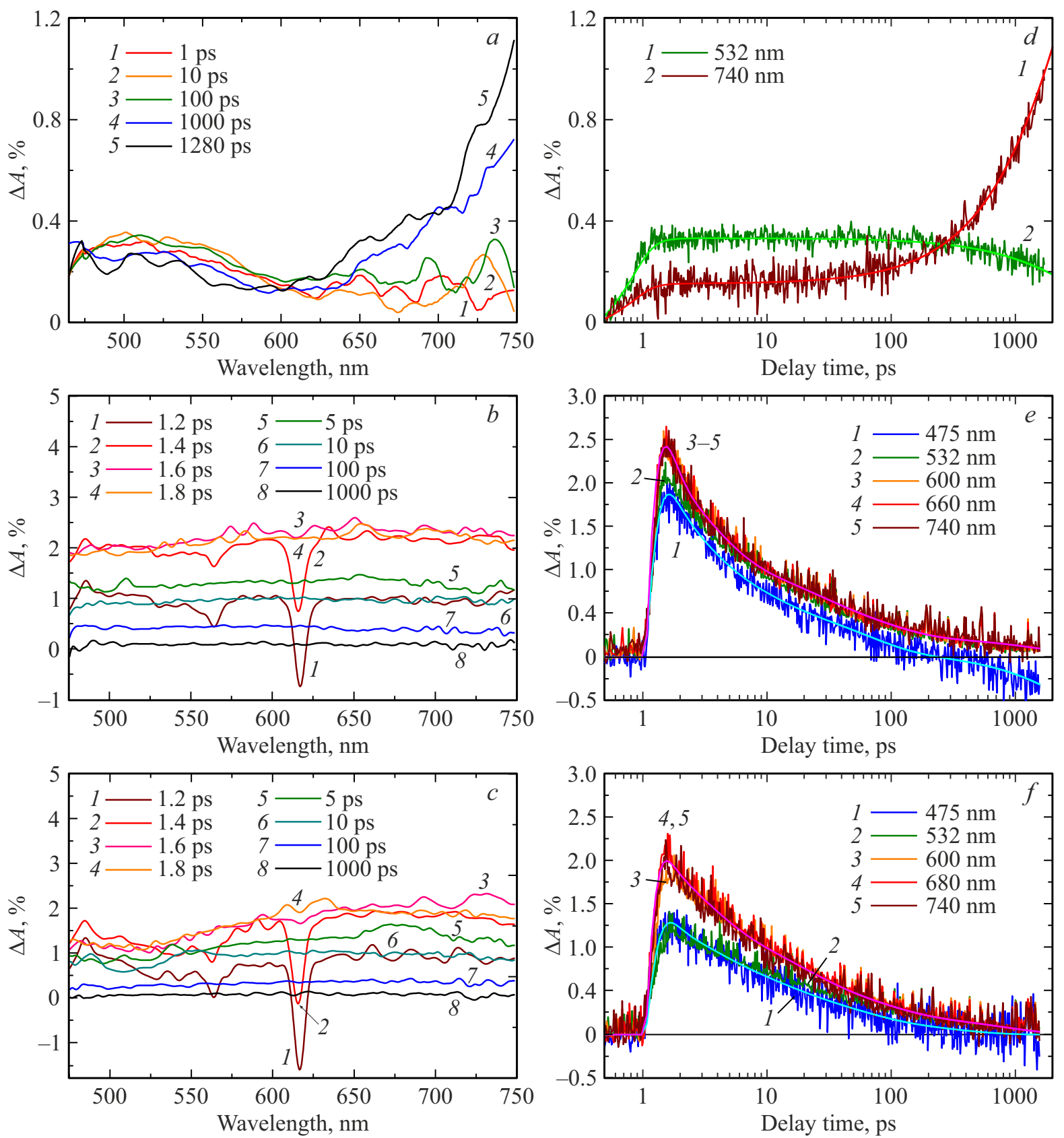

Рис. 4. Спектры $(a, b, c)$ и временной ход $(d, e, f)$ переходного поглощения в растворах $\mathrm{C}_{60}-$ бензол, $\mathrm{C}_{60}-\mathrm{NMP}$ (свежий) и $\mathrm{C}_{60}-\mathrm{NMP}$ (40 дней) соответственно. Спектры соответствуют временам задержки, указанным в легенде, временные зависимости длинам волн, указанным в легенде.

определения. Погрешность указанных величин, которая в отдельных случаях может достигать $20 \%$, в среднем может быть оценена в $15 \%$.

Видно, что различие во временах начальных процессов не столь велико. Различия начинаются со времен $\tau_{3}$ и $\tau_{4}$, которые принято приписывать тушению возбужденных состояний микроокружением нанокластеров [10]. В нашем случае это могут быть комплексы $\mathrm{C}_{60}-\mathrm{NMP}$, которые обнаруживаются в большом количестве в свежем растворе [14]. Известно также, что количество фракций мелких агрегатов (в несколько молекул) сильно падает после месяца хранения, уменьшая вместе с этим суммарную площадь поверхности наночастиц, т.е. концентрацию этих комплексов. Поэтому для старого раствора эти времена удлиняются, что видно при зондировании в области $475 \mathrm{~nm}$.

Более длительные времена, как правило, показывают время внутрисистемного переноса $\tau_{\text {isc. }}$. Например, в водном золе фуллерена это время наблюдалось равным $42.6 \mathrm{ps}$ [10]. Мы, однако, не можем однозначно 
Времена релаксации синглет-синглетного возбуждения (в ps) в растворе $\mathrm{C}_{60}-\mathrm{NMP}$ в зависимости от длины волны и возраста раствора

\begin{tabular}{c|c|c|c|c}
\hline \multirow{2}{*}{$\begin{array}{c}\text { Номер } \\
\text { процесса }\end{array}$} & \multicolumn{2}{|c|}{$475 \mathrm{~nm}$} & \multicolumn{2}{c}{$740 \mathrm{~nm}$} \\
\cline { 2 - 5 } & $\begin{array}{c}\text { Свежий } \\
\text { раствор }\end{array}$ & $\begin{array}{c}40 \text {-дневный } \\
\text { раствор }\end{array}$ & $\begin{array}{c}\text { Свежий } \\
\text { раствор }\end{array}$ & $\begin{array}{c}40 \text {-дневный } \\
\text { раствор }\end{array}$ \\
\hline$\tau_{1}$ & 0.21 & 0.21 & 0.165 & 0.14 \\
$\tau_{2}$ & 0.32 & 0.32 & 0.35 & 0.32 \\
$\tau_{3}$ & 1.8 & 2.5 & 2.7 & 2.5 \\
$\tau_{4}$ & 7 & 10 & 18 & 16 \\
$\tau_{5}$ & 60 & 50 & 80 & 75 \\
$\tau_{6}$ & 1100 & 300 & 1800 & 800 \\
$\tau_{7}$ & 5000 & - & - & -
\end{tabular}

определить, соответствуют ли времена $\tau_{5}$ и $\tau_{6}$ времени $\tau_{\text {isc }}$ для двух групп кластеров, сильно различающихся по размерам, или же они соответствуют временам $\tau_{0}$ и $\tau_{\text {isc }}$ для одной более или менее однородной группы кластеров большого размера, которая со значительной концентрацией образуется даже в свежем растворе [13]. Мы склоняемся ко второму варианту, поскольку мы не наблюдаем заметных изменений в амплитудах соответствующих экспонент при старении раствора.

На длине волны $740 \mathrm{~nm}$ на все указанные времена влияет неразличимый в наших условиях рост триплеттриплетного поглощения, поэтому их значения наблюдаются несколько большие, чем те, что получаются на $475 \mathrm{~nm}$. Дополнительное просветление с длинным хвостом $\left(\tau_{7}\right)$, наблюдаемое в свежем растворе на $475 \mathrm{~nm}$, может быть связано с включением реакции образования анион-радикала $\mathrm{C}_{60}^{\bullet-}$, с большой вероятностью происходящей в полярных растворителях [22].

Фотосесибилизация синглетного кислорода

Спектры ФЛ раствора $\mathrm{C}_{60}$ в NMP в ближней ИК области довольно сложные. На рис. 5, $a$ показан характерный спектр свежего раствора, полученный при возбуждении на $465 \mathrm{~nm}$, при котором люминесценция наиболее интенсивна.

Широкая полоса с максимумом на $1070 \mathrm{~nm}$, как мы полагаем, в основном сформирована анион-радикалом $\mathrm{C}_{60}^{\bullet-}$ с довольно богатым спектром флуоресценции [23]. Сопутствующие комплексы фуллерена с растворителем могут также вносить вклад. Спектр численно разделяется минимум на девять гауссовых контуров (рис. 5,a), среди которых хорошо заметна узкая полоса фосфоресценции СК с максимумом на $1276.0 \pm 0.5 \mathrm{~nm}$. Таким образом, её батохромный сдвиг, определяемый сильной полярностью $\mathrm{NMP}$, составляет $8 \mathrm{~nm}$. В неполярном $\mathrm{CCl}_{4}$ этот сдвиг меньше и составляет $\sim 4 \mathrm{~nm}[19]$.

Форма спектра ФЛ слабо меняется при изменении длины волны возбуждения. Эти изменения в основном состоят в более или менее синхронном изменении интенсивностей всех составляющих. Однако при возбуждении в красной области спектра доля некислородной
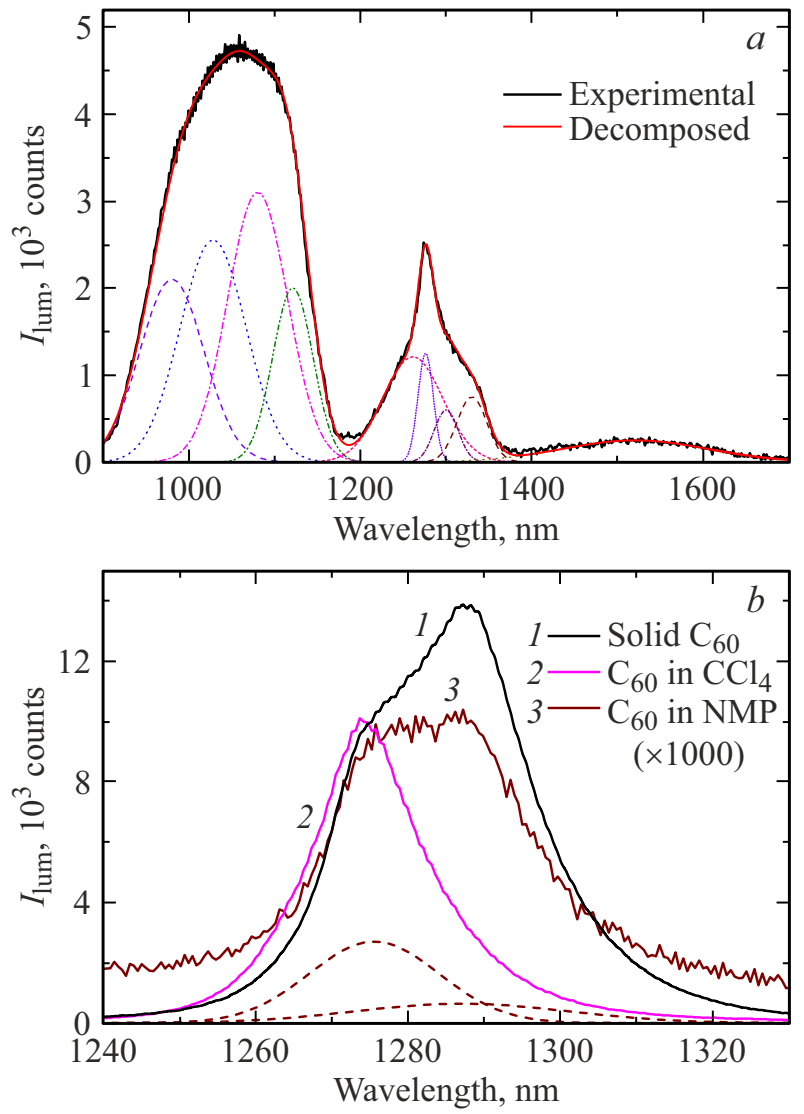

Рис. 5. Спектр ФЛ свежего раствора $\mathrm{C}_{60}$ в NMP (0.2 g/l) при возбуждении на $\lambda_{\mathrm{e}}=465 \mathrm{~nm}(a)$ и сравнение спектров фосфоресценции СК порошка твердого фуллерена $\mathrm{C}_{60}$, растворов $\mathrm{C}_{60}$ в $^{\mathrm{CCl}_{4}}(0.32 \mathrm{~g} / 1)$ и $\mathrm{C}_{60}$ в NMP $(0.2 \mathrm{~g} / 1)$, полученных в одинаковых условиях при возбуждении на $\lambda_{\mathrm{e}}=625 \mathrm{~nm}(b)$.

ФЛ падает, что дает возможность более точно оценить форму контура фосфоресценции СК и сравнить ее интенсивность с другими системами. Такое сравнение приведено на рис. $2, b$ с твердым порошком $\mathrm{C}_{60}$ и с раствором

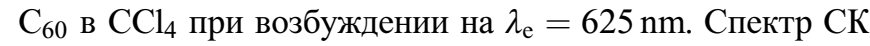
в растворе $\mathrm{C}_{60}$ в $\mathrm{NMP}$ получается шире, чем в $\mathrm{CCl}_{4}$, и также как и спектр от твердого $\mathrm{C}_{60}$ имеет компоненту с максимумом на $1287 \mathrm{~nm}$, соответствующую фосфоресценции СК, сорбированного твердофазным кристаллитом $\mathrm{C}_{60}$ [19]. После вычета сопутствующих полос (аналогично рис. 5,a) эти две компоненты фосфоресценции СК показаны на рис. 5, $b$ штриховыми кривыми. Их суммарная интегральная интенсивность относится к интенсивности полосы, наблюдаемой в растворе в $\mathrm{CCl}_{4}$, как $1 / 2750$.

Процесс ФС СК, схематично показанный на рис. 1, полностью определяется константами $k_{1}-k_{8}$. Поскольку за возбуждение состояния $T_{1}$ ответствен самый быстрый из процессов $k_{2}$ и $k_{3}$, соотношение скоростей которых сильно зависит от морфологии фуллерена, имеет смысл рассмотреть упрощенную схему, объединив процессы $k_{1}-k_{3}$ в один. Тогда мы будем оперировать лишь с двумя 
уровнями фуллерена (основным синглетным и триплетным), возбуждение которого будет характеризоваться константой $k_{\mathrm{ST}}$ :

$$
k_{\mathrm{ST}}=\frac{\sigma I}{h v} \phi_{\mathrm{T}},
$$

где $\sigma$ - сечение поглощения в основном состоянии, $I-$ интенсивность облучения на частоте $v, \phi_{\mathrm{T}}-$ квантовый выход триплетного состояния:

$$
\phi_{\mathrm{T}}=\frac{k_{3}}{k_{2}+k_{3}} .
$$

Тогда в условиях непрерывного облучения баланс населенностей $N_{1}, N_{3}-N_{6}$ уровней на рис. 1 определится следующей системой линейно независимых уравнений:

$$
\left\{\begin{array}{l}
k_{\mathrm{ST}} N_{1}-k_{4} N_{3}-k_{5} N_{3} N_{4}+k_{6} N_{1} N_{5}=0 \\
k_{5} N_{3} N_{4}-k_{6} N_{1} N_{5}-k_{7} N_{5}=0 \\
k_{7} N_{5}-k_{8} N_{6}=0 \\
N_{1}+N_{3}=N_{\mathrm{F}} \\
N_{4}+N_{5}+N_{6}=N_{\mathrm{O}}
\end{array}\right.
$$

где $N_{\mathrm{F}}$ - числовая плотность молекул фуллерена в растворе: $N_{\mathrm{F}}=2.7 \cdot 10^{17} \mathrm{~cm}^{-3}\left(\right.$ в $\left.\mathrm{CCl}_{4}\right)$ и $1.7 \cdot 10^{17} \mathrm{~cm}^{-3}$ (в NMP), а $N_{\mathrm{O}}$ - числовая плотность молекул кислорода в растворе. Последняя, согласно закону Генри, в воздухонасыщенном растворе должна составлять $21 \%$ от растворимости, т.е. $N_{\mathrm{O}}=1.6 \cdot 10^{18} \mathrm{~cm}^{-3}\left(\mathrm{~B} \mathrm{CCl}_{4}\right)$ и $4.1 \cdot 10^{17} \mathrm{~cm}^{-3}$ (в NMP). Таким образом, решая систему (6), легко рассчитать концентрацию СК в растворе $\mathrm{C}_{60}$ в $\mathrm{CCl}_{4}, N_{\mathrm{SO}}=N_{6}$, пользуясь коэффициентами, известными для мономолекулярного фуллерена [5]: $\phi_{\mathrm{T}}=1, \quad k_{4}=2.5 \cdot 10^{4} \mathrm{~s}^{-1}, \quad k_{5}=3.3 \cdot 10^{-12} \mathrm{~cm}^{3} \mathrm{~s}^{-1}$, $k_{6}=1 \cdot 10^{-17} \mathrm{~cm}^{3} \mathrm{~s}^{-1}, \quad k_{7}=1 \cdot 10^{8} \mathrm{~s}^{-1} . \quad$ Константа $k_{8}=k_{\mathrm{q}}=40 \mathrm{~s}^{-1}$ известна из наших измерений. В условиях нашего эксперимента (рис. 5, $b$ ) мы получаем $\quad N_{\mathrm{SO}}\left(\mathrm{CCl}_{4}\right)=2.32 \cdot 10^{17} \mathrm{~cm}^{-3}$, т. е. $14.8 \%$ от количества растворенного кислорода. В NMP эта концентрация, согласно нашим наблюдениям, будет в 2750 раз меньше, т.е. $N_{\mathrm{SO}}(\mathrm{NMP})=8.4 \cdot 10^{13} \mathrm{~cm}^{-3}$ или $0.02 \%$ от количества кислорода.

Из уравнений (6) непосредственно следует, что концентрация СК в растворе при непрерывном облучении

$$
N_{\mathrm{SO}}=\frac{k_{\mathrm{ST}}}{k_{8}} N_{\mathrm{F}}\left(1-\frac{N_{3}}{N_{\mathrm{F}}} \frac{k_{\mathrm{ST}}+k_{4}}{k_{\mathrm{ST}}}\right)=\frac{\sigma I}{h v} N_{\mathrm{F}} \phi_{\mathrm{T}} \phi_{\mathrm{SO}} \tau_{\mathrm{SO}} .
$$

Можно показать, что квантовый выход СК, $\phi_{\mathrm{SO}}$, введенный таким образом, не зависит от интенсивности облучения и определяется набором констант системы (6) [5]. Тогда, учитывая различие в концентрациях, сечениях поглощения, которые извлекаются из спектров (рис. 2), и определенных значений времен жизни СК (рис. 3) в двух растворителях, а также принимая для раствора $\mathrm{C}_{60}$ в $\mathrm{CCl}_{4}$ значения квантовых выходов $\phi_{\mathrm{T}}=0.93$ [24] и $\phi_{\mathrm{SO}}=0.96$ [4], определенные в толуольном и бензольном растворах соответственно, легко установить, что для раствора в NMP произведение квантовых выходов $\phi_{\mathrm{T}} \phi_{\mathrm{SO}}=0.74$. Погрешность этой величины мы оцениваем в $25 \%$, что связано с относительно большой возможной ошибкой разделения полос.

Результирующий квантовый выход получается неожиданно большим, учитывая наличие быстрого тушения состояния $S_{1}$, которое наблюдается в переходных спектрах. Объяснение этому противоречию может быть найдено в свете выдвинутого нами в предыдущем разделе предположения об интерпретации времен $\tau_{5}$ и $\tau_{6}$ как времен $\tau_{0}$ и $\tau_{\text {isc }}$ группы крупных однородных нанокластеров. Для мелких кластеров быстрое тушение уровня $S_{1}$ комплексами $\mathrm{C}_{60}-\mathrm{NMP}$, по-видимому, делает заселение триплета и соответственно генерацию СК неэффективными. Для крупных, в свете нашего предположения, мы имеем $\phi_{\mathrm{T}}=\tau_{5} /\left(\tau_{5}+\tau_{6}\right)=0.05$ для свежего раствора и 0.14 для 40-дневного раствора. Квантовый выход растет по мере укорочения времени внутрисистемного переноса при сохранении скорости тушения синглета, что, как мы предполагаем, происходит при увеличении размера частиц. Для крупных кластеров сечение поглощения отличается от молекулярного. Его можно получить в виде разности сечений экстинкции и рассеяния, которые определяются по формулам теории Ми:

$$
\begin{aligned}
& \sigma_{\text {ext }}=\frac{2 \pi}{k^{2}} \sum_{j=1}^{n}(2 j+1)\left(\operatorname{Re}\left\{a_{j}+b_{j}\right\}\right), \\
& \sigma_{\text {sca }}=\frac{2 \pi}{k^{2}} \sum_{j=1}^{n}(2 j+1)\left(\left|a_{j}\right|^{2}+\left|b_{j}\right|^{2}\right),
\end{aligned}
$$

где $k=2 \pi n_{\text {en }} / \lambda-$ волновое число $\left(n_{\text {en }}-\right.$ показатель преломления среды), а коэффициенты ряда рассеяния $a_{j}$ и $b_{j}$ рассчитываются через приведенный комплексный показатель преломления частицы $m=\tilde{n}_{\mathrm{P}} / n_{\mathrm{en}}$ и приведенный радиус частицы $x=2 \pi n_{\mathrm{en}} r / \lambda$ [25]. Для расчета сечений дисперсия комплексного показателя преломления фуллерена $\tilde{n}_{\mathrm{P}}$ была взята из работы [26]. Дисперсия показателя преломления NMP находилась преобразованием Крамерса-Кронига по известному распределению сечения поглощения в УФ области [27] и с привязкой статической части к известной величине $n_{\mathrm{D}}(\mathrm{NMP})=1.468$.

Расчет кинетики роста кластеров $\mathrm{C}_{60}$ в $\mathrm{NMP}$, выполненный в работе [13], показывает, что уже за несколько часов концентрация мономерной фракции падает в восемь раз, а средний радиус кластеров достигает $r=100 \mathrm{~nm}$. Этот радиус мы и приняли за характерный для свежего раствора. Для таких кластеров $n=9$ в суммах (8) достаточно для расчета сечения поглощения с точностью, превышающей $0.1 \%$. На длине волны $\lambda_{\mathrm{e}}=625 \mathrm{~nm}$ мы получаем значение $\sigma_{100 \mathrm{~nm}}=2.2763 \cdot 10^{-11} \mathrm{~cm}^{2}$. Концентрация нанокластеров пересчитывается из концентрации фуллерена в растворе в предположении сферической формы кластера и плотности твердого фуллерена $\rho_{\mathrm{F}}=1.46 \mathrm{~g} / \mathrm{cm}^{3}[28]$ 
и составляет $N_{\mathrm{Fnc}}=3.27 \cdot 10^{10} \mathrm{~cm}^{-3}$. С этими оценками сравнение интенсивностей фосфоресценции СК из pис. $5, b$ дает $\phi_{\mathrm{T}} \phi_{\mathrm{SO}}=0.23$, т.е. существенно меньше, чем получается без учета кластерного состава.

Дальнейшая эволюция кластеров в растворе $\mathrm{C}_{60}$ в NMP, прослеженная в работе [13], указывает на медленный рост их размеров до $\sim 500 \mathrm{~nm}$ на конечной стадии. Аналогичный расчет для радиуса $r=300 \mathrm{~nm}(\mathrm{c} n=11)$ дает $\sigma_{300 \mathrm{~nm}}=8.0535 \cdot 10^{-10} \mathrm{~cm}^{2}, N_{\mathrm{Fnc}}=1.21 \cdot 10^{9} \mathrm{~cm}^{-3}$ и $\phi_{\mathrm{T}} \phi_{\mathrm{SO}}=0.17$, что уже близко к $\phi_{\mathrm{T}}=0.14$, полученному нами для 40 -дневного раствора. Следует отметить, что дальнейшее увеличение радиуса наночастиц не приводит к уменьшению квантового выхода за счет чисто объемных эффектов. Поэтому должен существовать стационарный уровень эффективности ФС СК, достигаемый при старении раствора.

Кластерная природа ФС СК наблюдается и в зависимости концентрации СК от длины волны возбуждающего света. Интегральные интенсивности полосы фосфоресценции СК, выделенные из спектров ФЛ, записанных для свежего раствора и раствора после 5 месяцев хранения при разных светодиодах накачки, были пересчитаны в концентрацию СК в соответствии с установленной выше пропорцией. Эти концентрации были нормированы на величину плотности потока квантов на данной волне облучения и на время жизни СК в раствоpe: $\alpha_{\mathrm{eff}}=N_{\mathrm{SO}} h v /\left(I \tau_{\mathrm{SO}}\right)$. Получившаяся величина имеет смысл коэффициента эффективного (по отношению к ФС СК) поглощения раствора фуллерена и, согласно (7), равна произведению коэффициента поглощения на квантовые выходы: $\alpha_{\text {eff }}=\sigma N_{\mathrm{F}} \phi_{\mathrm{T}} \phi_{\mathrm{SO}}$. На рис. 6 точками показаны полученные спектральные зависимости эффективности ФС СК в терминах $\alpha_{\text {eff }}$ для свежего и старого растворов. Мы видим, что эти зависимости не воспроизводят спектральный ход коэффициента поглощения ни мономолекулярного фуллерена, ни раствора в NMP (рис. 2). Для свежего раствора $\alpha_{\text {eff }}$ имеет слабо выраженный максимум в области $450-460 \mathrm{~nm}$, а в старом - около $400 \mathrm{~nm}$.

Гораздо отчетливее прослеживается корреляция со спектрами поглощения наночастиц различного радиу$\mathrm{ca}$, рассчитанными по теории Ми и показанными на рис. 6 штриховыми линиями, которые имеют максимум в окрестности $450 \mathrm{~nm}$ и второй, более интенсивный максимум в УФ, который при укрупнении частиц приближается к $400 \mathrm{~nm}$. Кривой 1 на рис. 6 мы показали спектр, составленный из поглощения смеси молекул с наночастицами радиусом в $100 \mathrm{~nm}$ с соотношением фракций $8: 1$. Как нам представляется, этот спектр качественно описывает ход экспериментальной зависимости $\alpha_{\text {eff }}$ для свежего раствора. Спектру эффективного поглощения для старого раствора лучше всего соответствует спектр поглощения суспензии частиц с радиусом $300 \mathrm{~nm}$. Таким образом, вклад мономолекулярного фуллерена в ФС СК в этом случае минимальный.

Учет реального распределения наночастиц по размерам, а также уточнение дисперсии комплексного пока-

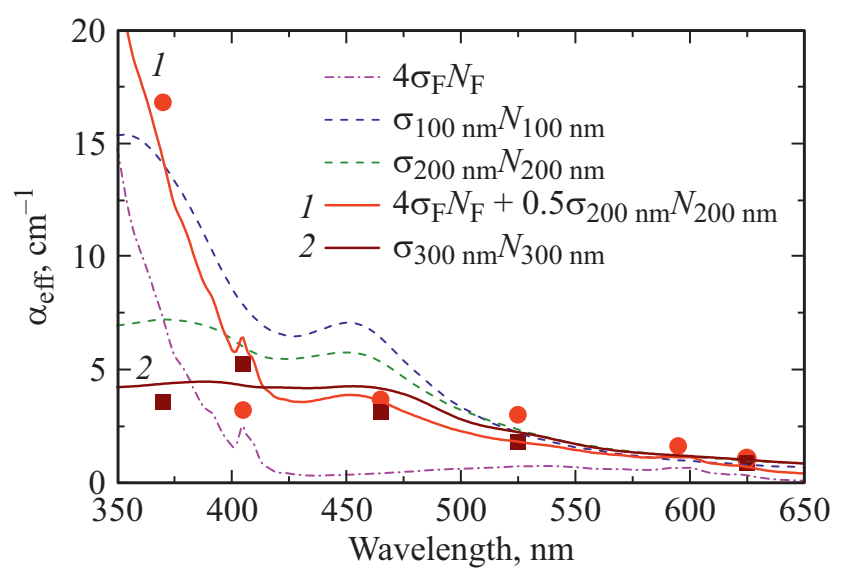

Рис. 6. Коэффициент эффективного поглощения свежего раствора $\mathrm{C}_{60}$ в NMP (круглые точки) и пятимесячного раствора (квадратные точки). Линиями показаны спектр мономолекулярного фуллерена (штрихпунктир), расчетные спектры коэффициента поглощения суспензии сферических наночастиц фуллерена в NMP c $r=100 \mathrm{~nm}$ и $200 \mathrm{~nm}$ (штриховые кривые), комбинированный спектр молекул и наночастиц (кривая 1) и расчетный спектр для наночастиц с $r=300 \mathrm{~nm}$ (кривая 2).

зателя преломления материала наночастиц фуллерена, который может отличаться от показателя преломления фуллерена в пленке, использованного в расчете, вероятно, воспроизвели бы наблюдаемые зависимости более точно. В силу обнаруженных корреляций спектра эффективности ФС СК со спектрами поглощения можно заключить, что мы не наблюдаем заметной зависимости квантового выхода СК от длины волны. Также не наблюдается его заметного изменения на фоне концентрационно-морфологических изменений при старении раствора.

\section{Заключение}

Проведенное исследование раствора $\mathrm{C}_{60}$ в NMP показало, что в переходных спектрах пикосекундного диапазона длительностей различаются шесть сверхбыстрых релаксационных компонент, относящихся к релаксации возбужденных синглетных состояний в первое возбужденное синглетное $S_{1}$, релаксации $S_{1}$ под влиянием микроокружения, а также более медленному внутрисистемному переносу на нижнее триплетное состояние $T_{1}$. В рамках предложенной идентификации времен квантовый выход триплетного состояния оценивается от $\phi_{\mathrm{T}}=0.05$ в свежем растворе до $\phi_{\mathrm{T}}=0.14$ в 40-дневном растворе.

В растворах обнаружена фосфоресценция СК. Совокупный квантовый выход СК, определенный с использованием раствора $\mathrm{C}_{60}$ в $\mathrm{CCl}_{4}$ в качестве репера, оказался равным 0.74 и независящим заметным образом от длины волны возбуждающего света. В то же время более низкая растворимость кислорода и сильное тушение СК $\boldsymbol{H}$-метилпирролидоном приводят к небольшой его кон- 
центрации в растворе, которая на три порядка меньше, чем в растворе $\mathrm{C}_{60}$ в $\mathrm{CCl}_{4}$ при прочих равных условиях.

При старении раствора квантовый выход остается того же порядка величины, несмотря на то, что форма спектральных зависимостей ФС СК-эффективного поглощения меняется особенно сильно в синей и УФ областях, указывая на различный состав агрегатов и исчезновение мономолекулярной фракции. Современные представления об эволюции агрегатов в растворе $\mathrm{C}_{60}$ в NMP находят в целом подтверждение и в нашем исследовании. Сохранение высокой эффективности ФС СК при старении раствора объясняется нами повышением квантового выхода триплетного состояния фуллерена в крупных агрегатах за счет ускорения внутрисистемного переноса и приближения его характерного времени к времени тушения синглета.

Мы полагаем, что обнаруженный и исследованный нами эффект ФС СК в воздухонасыщенном растворе $\mathrm{C}_{60}$ в NMP окажется интересным и полезным в областях традиционного применения СК: в биологии, медицине, лазерной технике и химическом производстве.

\section{Финансирование работы}

Настоящая работа выполнена при поддержке Китайской Академии наук (КАН): программы стратегических приоритетных исследований КАН (гранты №№ XDB16030700 и XDB43010303) и инициативы президента КАН по международным стипендиям (грант № 2021VTB0003).

\section{Конфликт интересов}

Авторы заявляют, что у них нет конфликта интересов.

\section{Список литературы}

[1] Dresselhaus M.S., Dresselhaus G., Eklund P.C. // J. Mater. Res. 1993. V. 8. P. 2054. doi 10.1557/JMR.1993.2054

[2] Белоусов В.П., Белоусова И.М., Будтов В.П., Данилов В.В., Данилов О.Б., Калинцев А.Г., Мак А.А // Оптический журн. 1997. Т. 64. № 12. С. 3.

[3] Макарова Т.Л. // ФТП. 2001. Т. 35. № 3. С. 257. doi $10.1134 / 1.1356145$

[4] Arbogast J.W., Darmanyan A.P., Foote C.S., Rubin Y., Diederich F.N., Alvarez M.M., Anz S.J., Whetten R.L. // J. Phys. Chem. 1991. V. 95. P. 11. doi 10.1021/j100154a006

[5] Белоусова И.М., Миронова Н.Г., Юрьев М.С. // Опт. и спектр. 2005. Т. 98. № 3. С. 390. doi 10.1134/1.1890511

[6] Багров И.В., Белоусова И.М., Киселев В.М., Кисляков И.М. // Оптический журн. 2019. Т. 86. С. 3. doi 10.1364/JOT.86.000066

[7] Abrahamse H., Hamblin M.R. // Biochem J. 2016. V. 473. N 4. P. 347. doi $10.1042 / \mathrm{BJ} 20150942$

[8] Innocenzi P., Stagi L. // Chem. Sci. 2020. V. 11. P. 6606. doi $10.1039 / \mathrm{d} 0 \mathrm{sc} 02658 \mathrm{a}$

[9] Белоусова И.М., Данилов О.Б., Муравьева Т.Д., Кисляков И.М., Рыльков В.В., Крисько Т.К., Киселев О.И.,
Зарубаев В.В., Сироткин А.К., Пиотровский Л.Б. // Оптический журн. 2009. Т. 76. № 4. С. 97. doi 10.1364/JOT.76.000243

[10] Clements A.F., Haley J.E., Urbas A.M., Kost A., Rauh R.D., Bertone J.F., Wang F., Wiers B.M., Gao D., Stefanik T.S., Mott A.G., Mackie D.M. // J. Phys. Chem. A. 2009. V. 113. P. 6437. doi 10.1021/jp8102518

[11] Белоусова И.М., Белоусов В.П., Киселев В.М., Муравьева Т.Д., Кисляков И.М., Сироткин А.К., Стародубщев А.М., Крисько Т.К., Багров И.В., Ермаков А.В. // Опт. и спектр. 2008. Т. 105. № 5. С. 770. doi 10.1134/S0030400X08110118

[12] Nakanishi I., Ohkubo K., Fujita S., Fukuzumi S., Konishi T., Fujitsuka M., Ito O., Miyata N. // J. Chem. Soc., Perkin Trans. 2002. V. 2. P. 1829 . doi $10.1039 / \mathrm{b} 206887 \mathrm{~g}$

[13] Tropin T.V., Jargalan N., Avdeev M.V., Kyzyma O.A., Eremin R.A., Sangaa D., Aksenov V.L. // J. Mol. Liquids. 2012. V. 175. P. 4. doi 10.1016/j.molliq.2012.08.003; Tpoпин T.B., Jargalan N., Авдеев М.B., Кизима O.A., Sangaа D., Аксенов В.Л. // ФТТ. 2014. Т. 56. № 1. С. 147. doi 10.1134/S1063783414010363

[14] Snegir S.V., Tropin T.V., Kyzyma O.A., Kuzmenko M.O., Petrenko V.I., Garamus V.M., Korobov M.V., Avdeev M.V., Bulavin L.A. // Appl. Surf. Sci. 2019. V. 481. P. 1566. doi 10.1016/j.apsusc.2019.03.168

[15] Khodayari M., Reinsberg P., Abd-El-Latif A.-E.-A. A., Merdon C., Fuhrmann J., Baltruschat H. // Chem. Phys. Chem. 2016. V. 17. P. 1647. doi 10.1002/cphc.201600005

[16] Murov S.L., Carmichael I., Hug G.L. Handbook of Photochemistry ( $2^{\text {nd }}$ ed.). 1993. NY: Marcel Dekker.

[17] Ebbesen T.W., Tanigaki K., Kurashima S. // Chem. Phys. Lett. 1991. V. 181. P. 501. doi 10.1016/0009-2614(91)80302-E

[18] Fraelich M.R., Weisman R.B. // J. Phys. Chem. 1993. V. 97. P. 11145. doi 10.1021/j100145a002

[19] Киселев В.М., Багров И.В. // Опт. и спектр. 2017. Т. 123. № 4. C. 543-554. doi 10.7868/S0030403417100105

[20] Hilton D.J. Ultrafast Pump-Probe Spectroscopy. / Ed. by Prasankumar R.P., Taylor A.J. Optical Techniques for Solid-State Materials Characterization. CRC Press, 2012.

[21] Ying Q., Marecek J., Chu B. // J. Chem. Phys. 1994. V. 101. P. 2665. doi $10.1063 / 1.467646$

[22] Beeby A., Eastoe J., Crooks E.R. // Chem. Commun. 1996. V. 8. P. 901. doi 10.1039/cc9960000901

[23] Kato T., Kodama T., Shida T. // Chem. Phys. Lett. 1993. V. 205. N 4-5. P. 405. doi 10.1016/0009-2614(93)87142-P

[24] Biczok L., Linschitz H., Walter R.I. // Chem. Phys. Lett. 1992. V. 195. N 4. P. 339. doi 10.1016/0009-2614(92)85613-F

[25] Bohren C.F., Huffman D.R. Absorption and Scattering of Light by Small Particles. John Wiley \& Sons, 1983; Борен K., Хафмен Д. Поглощение и рассеяние света малыми частицами. М.: Мир, 1986.

[26] Ren S.L., Wang Y., Rao A.M., McRae E., Holden J.M., Hager T., Wang K.A., Lee W.-T., Ni H.F., Selegue J., Eklund P.C. // Appl. Phys. Lett. 1991. V. 59. P. 2678. doi $10.1063 / 1.105907$

[27] Johnson E.A. UV Atlas of Organic Compounds 1966. V. 1. B10/4. NIST Chemistry WebBook. [Электронный pecypc] Режим доступа: https://webbook.nist.gov/cgi/ inchi? $\mathrm{ID}=\mathrm{C} 872504 \&$ Mask $=400 \# \mathrm{UV}$-Vis-Spec.

[28] Xiang H.-F., Xu Z.-X., Roy V.A.L., Che C.-M., Lai P.T. // Rev. Sci. Instr. 2007. V. 78. N 034104. doi 10.1063/1.2712932 